\title{
Effectiveness of Technetium-99 m monoclonal antibody scintigraphy for the detection of infectious focus in a case series in cirrhotic patients
}

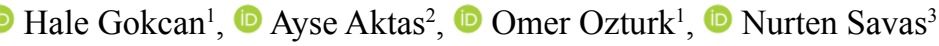 \\ ${ }^{I}$ Department of Gastroenterology, Ankara City Hospital, Ankara, Turkey; ${ }^{2}$ Department of Nucleer Medicine, Baskent University Faculty of Medicine, Ankara, \\ Turkey; ${ }^{3}$ Department of Gastroenterology, Baskent University Faculty of Medicine, Istanbul, Turkey
}

Dear Editor,

Infections are still responsible for overall morbidity and mortality despite increased knowledge about the microorganisms and antimicrobial treatment options. Clinicians need reliable methods for identification of the infection and inflammation in many clinical conditions, such as systemic bacterial infections, parasitic infestations or fever of unknown origin. Nuclear medicine plays an important role in the detection of the infection focus. It is used in varying degrees for identification of the infection focus in opportunistic infections, fever of unknown origin, postoperative infections, cardiovascular and central nervous system infections, osteomyelitis, and appendicitis.

An ample amount of scintigraphic methods have been found for the identification of infection or inflammation focus. Radiolabeled autologous leukocyte is used as the gold standard for identification of the infection with scintigraphic methods. ${ }^{[1]}$ Technetium (Tc)-99m-labeled antigranulocyte Fab fragment was used for the detection of the infection site in some patient groups (bone or soft tissue) and the method was shown to have high specificity and sensitivity.

Cirrhosis is a disease characterized by end-stage progressive hepatic fibrosis with widespread hepatic parenchymal destruction and replacement with collagenous scarring tissue, impaired normal hepatic lobular and vascular structure, and formation of regeneration nodules. Patients are exposed to many complications and expected survival is significantly shortened. Infection increases morbidity and mortality in the presence of ascites, hepatorenal syndrome, and hepatic encephalopathy. Although the diagnosis of infection is made based on clinical and biochemical findings, the infection focus (such as urinary tract, respiratory system, skin and intra-abdominal) and/or infectious agent cannot be detected with a physical examination, laboratory methods (urine culture, blood culture, chest graph, paracentesis culture and smear), imaging methods [ultrasound (USG), computed tomography, echography and magnetic resonance imaging (MRI) when needed] and the applied treatment method stay limited. Infection impairs prognosis in cirrhotic pa-

Received: March 10, 2020; Accepted: May 11, 2020; Available online: May 21, 2020

Corresponding author: Hale Gokcan; Ankara Sehir Hastanesi, Gastroenteroloji Klinigi, Ankara, Turkey

Phone: +90 31255260 00; e-mail: halesumer@yahoo.com

(c) (1) (8) OPEN ACCESS

(1) This work is licensed under a Creative Commons Attribution-NonCommercial 4.0 International License.

(C) Copyright 2020 by Hepatology Forum - Available online at www.hepatologyforum.org tients. Therefore, the most appropriate approach is early detection of the infection focus and the responsible microorganism and then initiating appropriate targeted treatment. Localizing the infectious site switches the empirical treatment to targeted treatment. This study was designated to investigate the effectiveness and reliability of Tc-99m monoclonal antibody scintigraphy (Leukoscan) in localizing infection focus.

\section{Materials and Methods}

The effectiveness of the Tc-99m-labeled antigranulocyte monoclonal antibody technique in the detection of infectious focus was investigated in cirrhotic patients. The study protocol was approved by the Baskent University Faculty of Medicine, IRB, approval number KA05/157, 20/09/2005. A total of eight Child C cirrhotic patients who were hospitalized for the presence of suspected infection based on history, clinical findings and elevated C-reactive protein (CRP) level and/or white blood cell (WBC) counts were included in this study. Focus of infection was investigated using X-ray, cultures (blood, urine, paracentesis) and/or imaging methods (USG, computed tomography, echocardiography and/or MRI) and localized in seven of the studied patients. Patients were injected 20 mCi Tc-99 monoclonal antibody (Leukoscan) after their signed informed consent. Whole-body scans and regional static images were obtained at hours 2 and 4 after $20 \mathrm{mCi}$ Tc-99 monoclonal antibody (Leukoscan) injection in eight Child $\mathrm{C}$ patients who were diagnosed with the infection.

\section{Results}

A total of eight cirrhotic patients ( 7 male and 1 female) were included in this study (4 cryptogenic, 2 hepatitis B infection, 1 hepatitis $C$ virus infection, 1 alcoholic). Mean age was $60.6 \pm 15.1$. Mean WBC count was $11500 / \mu \mathrm{L}, \mathrm{CRP}$ was $70 \mathrm{mg} / \mathrm{L}$. Based on anamnesis, physical examination, laboratory and imaging methods, urinary tract infection was detected in three patients (concurrent pulmonary infection in one patient, blood culture positivity in two patients), spontaneous bacterial peritonitis in three patients (concurrent abdominal skin infection in one patient), and pulmonary infection in one patient (Table 1). Infection site could not be detected in one patient despite the identification of infection through clinical and laboratory data (elevated CRP and WBC) (Table 1).

Eight patients underwent scintigraphy using Tc99m monoclonal antibody technique. The diagnostic focal increase in activity was not observed in any patients with Leukoscan scintigraphy. The non-specific, low-level diffuse activity was detected in five patients (Fig. 1). 


\begin{tabular}{ll}
\hline \multicolumn{2}{l}{ Table 1. Distribution of the infection foci } \\
\hline Patient & \multicolumn{1}{l}{ Infection sites } \\
\hline 1. & UTI \\
& Pulmonary infection \\
\hline 2. & UTI \\
& Blood culture positivity \\
3. & UTI \\
& Blood culture positivity \\
4. & SBP \\
& Abdominal skin infection \\
\hline 5. & SBP \\
6. & SBP \\
7. & Pulmonary infection \\
\hline 8. & No focus \\
\hline SBP: Spontaneous bacterial peritonitis; UTI: Urinary tract infection. \\
\hline
\end{tabular}

\section{Discussion}

In this study, we investigated the efficacy of Tc-99m-labeled antigranulocyte monoclonal antibody (Leukoscan) technique to determine the focus of infection in cirrhotic patients. However, we found that Leukoscan method is not an adequate method to determine infection focus in cirrhotic patients.

An ample amount of scintigraphic methods has been investigated for the identification of infection or inflammation focus in the recent 20 years. Effectiveness of radionuclide studies varies depending on the cause of the infection in patients who have infection but the focus cannot be detected. While the effectiveness of radionuclide studies and computed tomography was found to be similar for the discrimination of postoperative changes from infection in postoperative patients, radionuclide studies are in the foreground for elimination of the other causes in prosthesis infections. ${ }^{[2]}$

Many scintigraphic methods, like 99mTc-methylene diphosphonates (MDP), 67 Ga-citrate, indium-111 labeled oxyguinoline leukocyte (111-In oxin) and Tc-99m-hexamethylpropylamine (HMPAO)-labeled leukocyte are available for detection of infection focus. Radiolabeled autologous leukocytes have been used as the gold standard for the identification of the infection with scintigraphic methods. ${ }^{[1]} \mathrm{La}-$ beled leukocyte methods are the preferred method in immunocompromised patients. However, these methods have some difficulties (e.g., requiring laboratory conditions for in vitro labeling and the necessity for drawing blood and radiation exposure). In vivo labeling methods (peptides, antigranulocyte/antibody fragments) have been developed as alternative.

Use of radionuclide studies in infections has increased through discovery of labeling of the migrating inflammatory cells to the infection site. Uptake of the labeled leukocytes depends on chemotaxis (movement of the cells as response to chemical stimulation), number and type of the labeled cells and the number of the cellular component in inflammatory response. The method is mostly useful in infections with inflammatory response with neutrophil predominance (like bacterial infections) as most of the labeled leukocytes are neutrophils. The method is less successful in the infections where the other cellular responses are in the foreground rather than neutrophils.

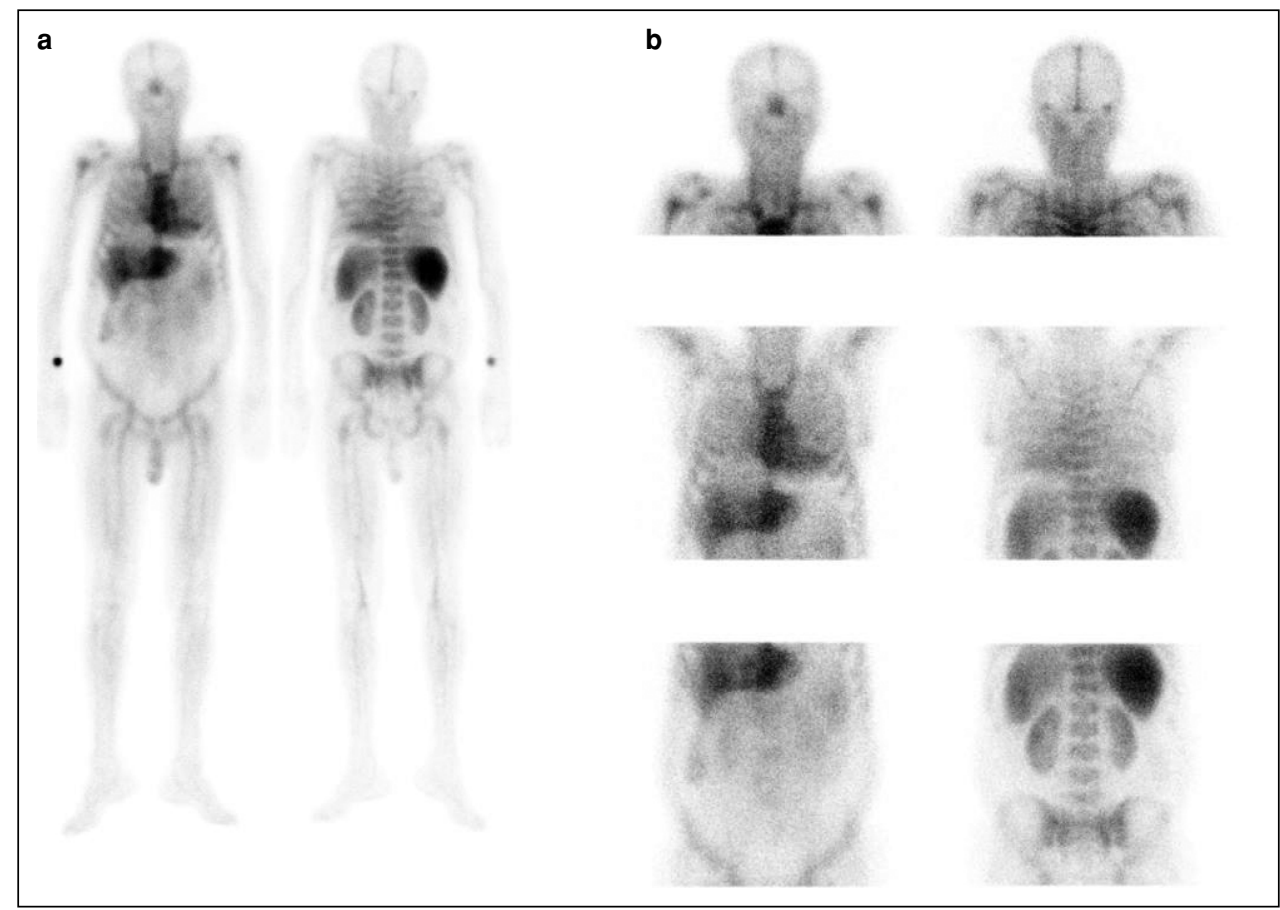

Figure 1. Whole body and static images obtained four hour after $20 \mathrm{mCi}$ Tc-99m Leukoscan injection in a cirrhotic patient with spontaneous bacterial peritonitis. It is evident from the images that the patient has ascites. There is only a slight increase in activity on the contour of abdominal region and a nonspecific slight increase in uptake on right upper-middle abdominal quadrant. 
Various alternative methods (like in vivo labeling methods) have been found considering the disadvantages observed in labeled leukocyte technique. Two types of Tc-99m-labeled antibody techniques are used. Although IgG1 antibody=intact murine antibody has a high sensitivity and specificity, its significant disadvantage is the $10-20 \%$ likelihood of allergic reaction development against human antimouse antibodies. ${ }^{[3]}$ Another option is using Fab fragment (IMMU$\mathrm{MN} 3=$ =leukoscan-sulesomab) instead of intact IgG1. ${ }^{[4]}$ Fab fragment accumulates in renal tissue, bone marrow, liver and spleen.

Tc99m-labeled antigranulocyte Fab fragment has been used for detection of infection site in some patient groups (bone and soft tissue) and the method has been shown to have a high sensitivity and specificity in these patient groups in various multicenter studies. As Fab fragments are small $(50 \mathrm{kd})$, images can be obtained rapidly 1-4 hours after the injection, they are uptaken by the lesion and imaging quality is high due to the low activity in the background. ${ }^{[4]}$

Tc-99m antigranulocyte Fab fragment has been shown to be a safe method also for the patient. Adverse effect was not observed or human antimouse antibody was not formed in humans. ${ }^{[5]}$ In the studies conducted with the patients who have the suspected soft tissue, vascular infection or bone infection, sensitivity and specificity of Tc-99m antigranulocyte Fab fragment was found higher than those of the other scintigraphic methods (In-111 oxin-labeled and Tc-99m HMPAO-labeled leukocyte). ${ }^{[5]}$ It is used in soft tissue infections and inflammations (like appendicitis), endocarditis and pulmonary infections. While sufficient blood supply and frequent acute infection in soft tissue and vascular structures are effective in successful results, poorer results may be obtained in osteomyelitis, periprosthetic bone infections as they become symptomatic in the advanced disease and manifested as chronic infections.

Tendency to bacterial infections are seen in cirrhotic patients due to intestinal disbiosis, increased intestinal bacterial translocation and cirrhosis-related immune dysfunction. Spontaneous bacterial peritonitis, urinary tract infection and subsequent pneumonia, cutaneous and soft tissue infections and bacteremia are frequent. ${ }^{[6]}$ Bacterial infections increase end-organ dysfunction and mortality in patients with decompensated cirrhosis. ${ }^{[7]}$ Early detection of the infectious agent and the focus reduces mortality and morbidity rates in cirrhotic patients by enabling the switch from the empirical to the targeted treatment in the early period.

Clinical suspicion of infection is of importance as the presentation of the infection and initial findings of the disease may be nonspecific and obscure in specific locations in this patient group. ${ }^{[6]}$ Therefore, the patients whose clinical condition deteriorates or who are suspected to have an infection should be comprehensively evaluated in the early period. However, CRP and procalcitonin levels may be low despite the presence of infection. ${ }^{[8]}$ Reduction in neutrophils, $\mathrm{T}$ and $\mathrm{B}$ cell count, impairment in neutrophil functions, reduction in phagocytosis and chemotaxis (mobilization) may also be seen. ${ }^{[9,10]}$ The low sensitivity and specificity of the conventional parameters in infection diagnosis in cirrhotic patients and presence of cirrhosisassociated immune dysfunction make the detection of the infection and diagnosis difficult. Therefore, novel methods are required for the identification of the infection focus. ${ }^{[6]}$ Leukoscan method is known to have a high sensitivity and specificity in the identification of infec- tion focus in some patient groups (such as bone, soft tissue).$^{[5]}$ Based on these studies, investigation of the effectiveness of Leukoscan scintigraphy in the detection of infection focus in patients with cirrhosis and infection was planned. This study was conducted with the aim of early detection of infectious agent and focus in CLD patients, reducing morbidity and mortality by enabling the switch from empirical to targeted treatment. This may be accepted as a preliminary study conducted with limited number of patients. It was seen that diagnostic focal activity uptake was not observed for infection focus with leukoscan method in cirrhotic patients.

The scintigraphic method is useful in localizing infections having an inflammatory response with neutrophil predominance as vast majority of the labeled leukocytes are neutrophils. Uptake of the labeled leukocytes is dependent on the normal chemotaxis, number and type of the labeled cells in labeled leukocyte scintigraphy. In our study, Leukoscan method was considered not to be effective in detection of infection in cirrhotic patients due to the presence of cirrhosis-related immune dysfunction; reduction in number and function of neutrophils. Because this study is a pilot study in cirrhosis, patients and the number of patients are low, negative results may be obtained. More accurate results can be obtained with more patients and the participation of the center.

Peer-review: Externally peer-reviewed.

Author Contributions: Concept - HG, AA, OO; Design - HG, AA, NS; Supervision - HG, AA, NS; Materials - HG, AA, OO; Data Collection and/or Processing - HG, AA, OO; Analysis and/or Interpretation - HG, AA, OO; Literature Search - HG, OO; Writing - HG, AA; Critical Reviews - HG, AA, NS.

Conflict of Interest: The authors do not have any conflict of interest.

Financial Disclosure: The authors declared that this study has received no financial support.

\section{References}

1. Bleeker-Rovers CP, Boerman OC, Rennen HJ, Corstens FH, Oyen WJ. Radiolabeled compounds in diagnosis of infectious and inflammatory disease. Curr Pharm Des 2004;10(24):2935-2950.

2. Erba PA, Sollini M, Conti U, Bandera F, Tascini C, De Tommasi SM, et al. Radiolabeled WBC scintigraphy in the diagnostic workup of patients with suspected device-related infections. JACC Cardiovasc Imaging 2013;6(10):1075-1086.

3. Goldenberg DM, Ford EH, Lee RE. Initial clinical imaging results with a new Tc-99m-antibody labeling method. J Nucl Med 1989;30:809.

4. Goldenberg DM, Larson SM. Radioimmunodetection in cancer identification. J Nucl Med 1992;33(5):803-814.

5. Becker W, Palestro CJ, Winship J, Feld T, Pinsky CM, Wolf F, et al. Rapid imaging of infections with a monoclonal antibody fragment (LeukoScan). Clin Orthop Relat Res 1996;(329):263-272.

6. Jalan R, Fernandez J, Wiest R, Schnabl B, Moreau R, Angeli P, et al. Bacterial infections in cirrhosis: a position statement based on the EASL Special Conference 2013. J Hepatol 2014;60(6):1310-1324.

7. Arvaniti V, D’Amico G, Fede G, Manousou P, Tsochatzis E, Pleguezuelo $\mathrm{M}$, et al. Infections in patients with cirrhosis increase mortality fourfold and should be used in determining prognosis. Gastroenterology 2010;139(4):1246-1256.

8. Bota DP, Van Nuffelen M, Zakariah AN, Vincent JL. Serum levels of C-reactive protein and procalcitonin in critically ill patients with cirrhosis of the 
liver. J Lab Clin Med 2005;146(6):347-351.

9. Tritto G, Bechlis Z, Stadlbauer V, Davies N, Francés R, Shah N, et al. Evidence of neutrophil functional defect despite inflammation in stable cirrhosis. J Hepatol 2011;55(3):574-581.
10. Doi H, Iyer TK, Carpenter E, Li H, Chang KM, Vonderheide RH, et al. Dysfunctional B-cell activation in cirrhosis resulting from hepatitis $\mathrm{C}$ infection associated with disappearance of CD27-positive B-cell population. Hepatology 2012;55(3):709-719. 